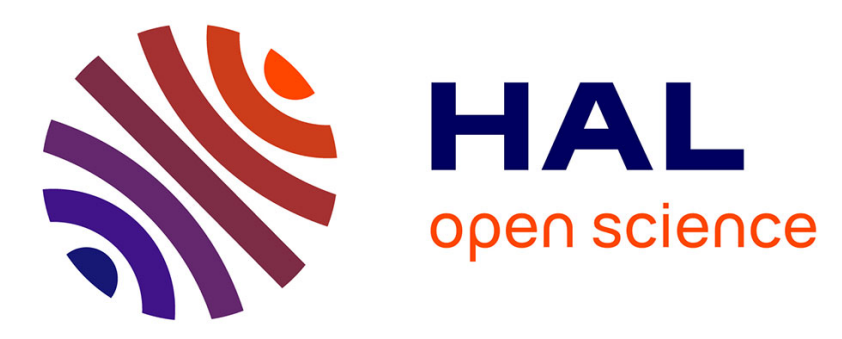

\title{
A Novel Model-Based Robust Super-Twisting Sliding Mode Control of PKMs: Design and Real-Time Experiments
}

\author{
Hussein Saied, Ahmed Chemori, Maher El Rafei, Clovis Francis
}

\section{To cite this version:}

Hussein Saied, Ahmed Chemori, Maher El Rafei, Clovis Francis. A Novel Model-Based Robust Super-Twisting Sliding Mode Control of PKMs: Design and Real-Time Experiments. IROS 2021 - IEEE/RSJ International Conference on Intelligent Robots and Systems, Sep 2021, Prague, Czech Republic. pp.8029-8035, 10.1109/IROS51168.2021.9636168 . lirmm-03343014v2

\section{HAL Id: lirmm-03343014 https://hal-lirmm.ccsd.cnrs.fr/lirmm-03343014v2}

Submitted on 9 Oct 2021

HAL is a multi-disciplinary open access archive for the deposit and dissemination of scientific research documents, whether they are published or not. The documents may come from teaching and research institutions in France or abroad, or from public or private research centers.
L'archive ouverte pluridisciplinaire HAL, est destinée au dépôt et à la diffusion de documents scientifiques de niveau recherche, publiés ou non, émanant des établissements d'enseignement et de recherche français ou étrangers, des laboratoires publics ou privés. 


\title{
A Novel Model-Based Robust Super-Twisting Sliding Mode Control of PKMs: Design and Real-Time Experiments
}

\author{
Hussein Saied ${ }^{1,2}$, Ahmed Chemori ${ }^{1}$, Maher El Rafei ${ }^{2}$ and Clovis Francis ${ }^{2}$
}

\begin{abstract}
In this paper, a new robust model-based supertwisting algorithm is proposed as a control solution for parallel kinematic manipulators (PKMs). The conventional supertwisting algorithm for robot manipulators has the structure of a computed-torque control which can be sensitive to measurement noise. This issue may deteriorate the dynamic performance of the manipulator and reduce its robustness towards changes in the operating conditions. The proposed approach, relying on the desired trajectory, is more computationally efficient and more robust. It includes a feedforward dynamic compensator, the super-twisting feedback control, and a feedback stabilizing term. As a validation, real-time experiments have been conducted on a 5-DOF redundantly actuated PKM. Several scenarios have been tested including nominal case and the robustness towards speed variations. The relevance of the proposed control solution is proved through the improvement of the tracking performance at different dynamic operating conditions.
\end{abstract}

\section{INTRODUCTION}

Over the last few decades, the interest about Parallel Kinematic Manipulators (PKMs) has increased thanks to their distinguished features. In contrast to their serial counterparts, parallel manipulators offer more stiffness, better accuracy, high-speed capabilities, and a higher payload-toweight ratio. However, they still suffer from some drawbacks such as the limited workspace and the complex singularities behavior. The control design of PKMs is a key factor in obtaining satisfactory high dynamic performances [1]. Parallel manipulators are known by their high nonlinearities, coupled actuation, uncertainties, and actuation redundancy, which make their control task more difficult and challenging [2]. In the literature, a vast number of control solutions have been proposed and experimented on parallel manipulators [3] [4] [5].

On the one hand, the non-model-based control solutions, which are simple to be implemented, do not take into account the dynamics of the manipulator [6], [7]. Thus, the dynamic performance can be deteriorated leading sometimes to instability at high-speed motions. On the other hand, the model-based control solutions consider, fully or partially, the dynamics of the manipulator in their design, compensating for the high effect of the nonlinear dynamics [8], [9], [10], [11], [12]. As a result, model-based controllers can provide much higher performance and robustness towards nonlinearity variation compared to non-model-based controllers.

Sliding Mode Control (SMC) is a powerful tool against model uncertainties, unknown and/or time varying plant

\footnotetext{
${ }^{1}$ Hussein Saied and Ahmed Chemori are with LIRMM, University of Montpellier, CNRS, Montpellier, France \{saied, chemori\}@lirmm. fr

${ }^{2}$ Hussein Saied, Maher El Rafei and Clovis Francis are with CRSI, Lebanese University, Beirut, Lebanon \{maher.elrafei, cfrancis\}@ul.edu.lb
}

parameters, as well as parasitic dynamics [13]. Its control objective is to allow the convergence of a sliding variable, composed of the system states, to zero, and thus converge the system states to the sliding surface in a finite time and remain on it, allowing the state trajectories converge towards zero. SMC can provide the controlled system a high robustness towards disturbances thanks to the theoretical exact compensation of the bounded matched disturbances. However, standard SMC produces a discontinuous control signal which is not adequate with real-time implementation. Several techniques have been investigated in the literature to avoid or reduce this high-frequency switching, known as chattering phenomena, such as Quasi-SMC, integration of sign function, continuous high-order SMC [13], etc.

Furthermore, second-order SMC algorithms are able to achieve finite-time convergence of the sliding variable and its derivative. It can ensure a quadratic precision of the convergence with respect to the sliding output as well as the sliding surface is no longer needed (Twisting and Terminal controllers) [14], [15]. The second-order Super-Twisting SMC (ST-SMC) algorithm has been proposed and developed, resulting in an exact finite-time convergence of the sliding variable and its derivative, as well as a high accurate asymptotic convergence of the states, and a continuous control signal [16].

Decentralized SMC has been applied to parallel manipulators such that none of the dynamic parts of the manipulator does appear in the control law [17], [18]. An adaptive terminal sliding mode control has been proposed in [19] for parallel manipulators. To the best of the authors knowledge, while ST-SMC approach has never been applied to parallel manipulators, it has some other robotic applications. Those applications include under-actuated robotic systems [20], attitude tracking problem of quadrotors [21], and mobile robots [22]. Furthermore, a variable-gain ST-SMC version has been proposed for a robotic arm in [23] allowing to compensate for a larger class of perturbations than the conventional ST-SMC.

In this paper, a novel model-based ST-SMC is proposed for parallel manipulators as an extension of the original secondorder ST algorithm [16]. The proposed control structure formed by a dynamic feedforward term, a feedback supertwisting term, and a stabilizing feedback term, can be more adequate for real-time implementations. Moreover, this control scheme can be easily applied to the general class of robotic manipulators whose dynamics is described by (4).

It could be less sensitive to measurements noise that may deteriorate the dynamic performance and stimulate the chattering effect. Experimental tests are conducted on a fiveDegrees-Of-Freedom (5-DOF) redundant parallel manipulator, called SPIDER4 robot, showing an improved dynamic 


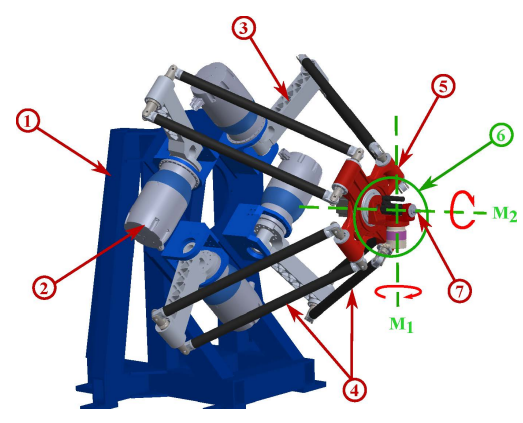

Fig. 1. A CAD view of SPIDER4 PKM, including (1) : Fixed-base, (2): Actuators, (3): Rear-arms, (4): Forearms, (5): Travelling plate, (6): Serial wrist mechanism, (7): Spindle.

performance and a more robustness towards disturbances and changes in the operating conditions.

The rest of the paper is organized as follows. Section II introduces a brief description of the structure and modeling of SPIDER4 robot. The proposed control solution is introduced in section III. The real-time experimental results are presented and discussed in section IV. Section V sums up the main drawn conclusions and proposes future directions of the present work.

\section{SPIDER4 ROBOT: DESCRIPTION AND MODELING}

\section{A. Description of SPIDER4 robot}

SPIDER4 is a Delta-like 5-DOF redundantly actuated parallel manipulator, designed at LIRMM for machining applications [24]. Fig. 1 shows a CAD-view of SPIDER4 PKM and its main components. It consists of a fixed-base linked to a moving platform (nacelle) through four kinematic chains. Each kinematic chain is composed of an actuator (rotational motor), a rear-arm, and a forearm. The overall assembly allows the nacelle to perform in three translational motions $x, y$ and $z$. An additional independent serial wrist mechanism (actuated by two motors) is attached to the nacelle, offering two more rotational movements for the machining spindle around the axes of the motors $M_{1}$ and $M_{2}$ (cf. Fig. 1). In this work, we are mainly interested in the control of the parallel structure (Delta-like positioning mechanism) of SPIDER4 robot.

\section{B. Kinematics of SPIDER4 robot}

As previously mentioned, in our modeling and control developments, we are concerned only with the parallel Deltalike positioning structure, the orientation of the spindle is kept constant. Consider $X=[x, y, z]^{T}$ as the Cartesian position vector of the nacelle center and $q=\left[q_{1}, q_{2}, q_{3}, q_{4}\right]^{T}$ as the joint position vector. The kinematic model of SPIDER4 robot describes the geometric relationship between the actuated joint angles and the Cartesian position of the traveling-plate.

The Jacobian matrix provides the differential kinematic relationship between the Cartesian- and joint-space velocities as follows: $\dot{X}=J \dot{q}$, where $\dot{X}, \dot{q}$ are the Cartesian and joint velocity vectors respectively. $J$ is the direct Jacobian matrix.

The kinematic model is used to calculate the range of errors we can get at Cartesian space, since the manipulator is a translational robot. All the segments' lengths have been measured after manufacturing the robot and adjusted accordingly without calibrating the kinematic model. The offset error effects are canceled after derivation. Knowing that the joint and Cartesian errors are linked through the Jacobian matrix, the kinematic calibration may not change that much the range of errors.

\section{Dynamics of SPIDER4 robot}

The dynamic model of SPIDER4 robot is introduced in this section, based on the virtual work principle described in [25]. As commonly supposed for Delta-like PKMs, the following two assumptions are considered for a simplification purpose:

Assumption 1: Both dry and viscous frictions in all passive and active joints are neglected.

Assumption 2: The forearms' mass is split up into two point-masses, the first one is added to the mass of the reararms, while the second one is considered with the mass of the traveling-plate.

On the one hand, the traveling-plate dynamics can be represented by the torque contributions at the joints side of the gravitational and inertial forces acting on the travelingplate as follows:

$$
\Gamma_{G_{t p}}=-J^{T} M_{t p} G \quad, \quad \Gamma_{F_{t p}}=J^{T} M_{t p} \ddot{X}
$$

where $M_{t p}=\operatorname{diag}\left\{m_{t p}, m_{t p}, m_{t p}\right\}$ is the total mass matrix including the mass of the nacelle, the payload lifted by the nacelle (the wrist including the two motors and the spindle), and the half-masses of the forearms, $\ddot{X} \in \mathbb{R}^{3}$ is the Cartesian acceleration vector and $G=[0, g, 0]^{T}$ represents the gravity vector with $g=9.81 \mathrm{~m} / \mathrm{s}^{2}$ being the gravity acceleration.

On the other hand, the dynamics of the rear-arms, from the joint side, comprise (i) the torque generated by the actuators $\Gamma \in \mathbb{R}^{4}$, (ii) the torque contribution of the gravitational force acting on the rear-arms $\Gamma_{G_{a r m}} \in \mathbb{R}^{4}$, and (iii) the inertial contribution due to the rear-arms' acceleration $\Gamma_{\text {arm }} \in \mathbb{R}^{4}$. After some geometric considerations, one can obtain the torque contribution of the gravitational effects on the reararms as follows:

$$
\Gamma_{G_{a r m}}=-g M_{r \alpha} \operatorname{Cos}(q)
$$

where $M_{r \alpha}$ is a geometric constant and $\operatorname{Cos}(q) \triangleq$ $\left[\cos \left(q_{1}\right), \cos \left(q_{2}\right), \cos \left(q_{3}\right), \cos \left(q_{4}\right)\right]^{T}$. The torque contribution of the inertial force acting on the rear-arms can be defined as follows:

$$
\Gamma_{\text {arm }}=I_{a r m} \ddot{q}
$$

where $I_{\text {arm }} \in \mathbb{R}^{4 \times 4}$ is a diagonal inertia matrix including the inertia of the actuators, the rear-arms and the half-masses of the forearms with respect to the actuators' rotation axes. $\ddot{q} \in \mathbb{R}^{4}$ is the acceleration vector in joint space.

Finally, the inverse dynamic model of SPIDER4 PKM can be formulated using the virtual work principle as follows:

$$
\Gamma(t)=M(q) \ddot{q}+C(q, \dot{q}) \dot{q}+G(q)
$$

where $M(q)=I_{a r m}+J^{T} M_{t p} J$ is the total mass and inertia matrix of the robot, $C(q, \dot{q})=J^{T} M_{t p} \dot{J}$ is the Coriolis and centrifugal forces matrix, $G(q)=-\Gamma_{G_{t p}}-\Gamma_{G_{a r m}}$ is the gravitational forces vector, and $\Gamma(t)$ is the control input vector. The main dynamic parameters of SPIDER4 parallel robot are summarized in Table I. 


\section{PROposed MOdEL-BASEd SUPER-TWISTING SMC}

\section{A. Motivation}

If we consider the external disturbances, the dynamic model can be rewritten as:

$$
M(q) \ddot{q}+C(q, \dot{q}) \dot{q}+G(q)+\Gamma_{d}=\Gamma
$$

where $\Gamma_{d} \in \mathbb{R}^{n}$ represents the vector of external disturbances, uncertainties, and non-modeled dynamics. Assuming that $\Gamma_{d}$ is bounded, the conventional ST-SMC algorithm that can be designed for such type of systems can be expressed as follows [16], [26]:

$$
\begin{aligned}
\Gamma & =M(q)\left(\ddot{r}+\Gamma_{S T-S M C}\right) \\
\Gamma_{S T-S M C} & =k_{1}|s|^{\frac{1}{2}} \operatorname{sign}(s)+w \\
\dot{w} & =k_{2} \operatorname{sign}(s)
\end{aligned}
$$

where $\ddot{r}=\ddot{q}_{d}+\lambda \dot{e}$ with $\lambda$ being a positive feedback gain, the tracking error is defined as $e=q_{d}-q$, and $s=\dot{e}+\lambda e$ being the sliding surface. One of the main limitations of this control structure is the lack of some parts of the model dynamics (actually including only the inertia matrix) which may decrease the dynamic performance of the parallel robot.

The control design of the ST-SMC approach taking into account the nonlinear dynamics within a computed-torque formulation can be expressed as follows [21], [23]:

$$
\Gamma=M(q)\left(\ddot{r}+\Gamma_{S T-S M C}\right)+C(q, \dot{q}) \dot{q}+G(q)
$$

This computed-torque control based on the ST-SMC algorithm needs a well and precise knowledge of the dynamic model to obtain satisfactory tracking performances. Relying on measured signals to compute the dynamic model in an online form can also make the controller more sensitive to noise measurements, decreasing the global performance and increasing the chattering effect. Furthermore, it has been shown in [27] that computed-torque control is unable to cope well with modeling errors.

To avoid all the above issues, we propose to replace the computed-torque with a feedforward term having at the end a super-twisting feedforward sliding mode control approach. The feedforward dynamic term, relying on the desired trajectories instead of the measured ones, can be much more efficient in terms of computation cost, since it can be computed offline and stored to be used online within the control. Also, it is less sensitive measurement noises providing then a better performance and a less chattered signal.

TABLE I

SUMMARY OF THE MAIN DYNAMIC PARAMETERS OF SPIDER4 PKM.

\begin{tabular}{ccc}
\hline Parameter & Description & Value \\
\hline$L$ & Rear-arm length & $535 \mathrm{~mm}$ \\
$l$ & Forearm length & $1100 \mathrm{~mm}$ \\
$m_{r}$ & Rear-arm mass & $17.6 \mathrm{~kg}$ \\
$m_{f}$ & Forearm mass & $4.64 \mathrm{~kg}$ \\
$m_{t p}$ & Total traveling-plate mass & $51.54 \mathrm{~kg}$ \\
$I_{\text {arm }}$ & Rear-arm inertia & $1.69 \mathrm{~kg} \cdot \mathrm{m}^{2}$ \\
$I_{\text {act }}$ & Actuator inertia & $2.23 \times 10^{-3} \mathrm{~kg} \cdot \mathrm{m}^{2}$ \\
\hline
\end{tabular}

\section{B. Control design}

This section provides a step-by-step derivation of the proposed feedforward ST-SMC algorithm. The standard sliding surface for a super-twisting SMC algorithm can be defined as follows:

$$
s=\dot{e}+\Lambda e
$$

with $\Lambda$ being a positive definite diagonal matrix of feedback gains for each axis.

Combining the defined sliding surface (8) and the dynamic model (5) leads to the following equation:

$$
M(q)\left(\ddot{q}_{d}-\dot{s}+\Lambda \dot{e}\right)+C(q, \dot{q}) \dot{q}+G(q)+\Gamma_{d}=\Gamma
$$

Let us now define an auxiliary reference velocity trajectory $\dot{r}=\dot{q}_{d}+\Lambda e$ shifted from the actual desired one by $\Lambda \dot{e}$. Then, (9) can be re-written as follows:

$$
M(q) \ddot{r}-M(q) \dot{s}+C(q, \dot{q}) \dot{q}+G(q)+\Gamma_{d}=\Gamma
$$

where $\ddot{r}$ is the corresponding shifted desired acceleration. The sliding surface dynamics can be obtained from (10) as follows:

$$
\dot{s}=M^{-1}(q)\left(-\Gamma+M(q) \ddot{r}+C(q, \dot{q}) \dot{q}+G(q)+\Gamma_{d}\right)
$$

Then, the control input $\Gamma$ can be chosen to have an exact compensation for the nonlinearities of the dynamic model as well as for the external disturbance term. The conventional model-based super-twisting SMC control can be defined as follows:

$$
\begin{aligned}
& \Gamma=M(q)\left(\ddot{r}+K_{2}|s|^{\frac{1}{2}} \operatorname{sign}(s)+w\right)+C(q, \dot{q}) \dot{q}+G(q) \\
& \dot{w}=K_{3} \operatorname{sign}(s)
\end{aligned}
$$

where $K_{2}, K_{3}$ are two positive definite diagonal matrices. Note that the control law in (12) is in the form of computedtorque control based on the super-twisting algorithm.

The proposed feedforward ST-SMC algorithm includes three main parts: (i) the feedforward term, (ii) the supertwisting algorithm, and (iii) a feedback term added to insure the stability of the closed-loop system. The expression of the proposed control law is given as follows:

$$
\begin{gathered}
\Gamma=M\left(q_{d}\right) \ddot{q}_{d}+C\left(q_{d}, \dot{q}_{d}\right) \dot{q}_{d}+G\left(q_{d}\right) \\
+K_{1} s+K_{2}|s|^{\frac{1}{2}} \operatorname{sign}(s)+w \\
\dot{w}=K_{3} \operatorname{sign}(s)
\end{gathered}
$$

where $K_{1}$ is a positive definite diagonal matrix of the feedback control gains.

Let us now assume that the desired dynamic terms compensate for the nonlinearities of the system, or the difference between the desired and the exact dynamics is bounded within a small range [28]. Moreover, the super-twisting term is assumed to compensate theoretically for the disturbances. Then, the closed-loop error dynamics can be obtained after substituting the control law (13) in the sliding surface dynamics (11) leading to:

$$
\left(I-\Lambda+K_{1} M^{-1}(q)\right) \dot{e}=-\Lambda\left(I+K_{1} M^{-1}(q)\right) e
$$


From (14), if $\Lambda$ and $K_{1}$ are chosen such that the two matrices $\left(I-\Lambda+K_{1} M^{-1}(q)\right)$ and $\Lambda\left(I+K_{1} M^{-1}(q)\right)$ are positive definite, then the error can converge exponentially to zero as time goes to infinity. Thus, the stability of the system can be guaranteed under the proposed controller.

\section{REAL-TIME EXPERIMENTAL RESULTS}

In this section, the obtained experimental results of the conventional Computed-Torque ST-SMC (CT-ST-SMC) algorithm (12) and the proposed FeedForward ST-SMC (FFST-SMC) algorithm (13) are presented and compared in comparison with a standard PID with computed FeedForward (PIDFF) controller.

\section{A. Experimental platform and implementation issues}

1) Experimental platform: The experimental platform of SPIDER4 robot is shown in Fig. 2. The parallel structure of SPIDER4 robot consists of four $\mathrm{TPM}^{+}$high torque rotary motors. A gearbox of a gear ratio of 22 is merged seamlessly to the motor forming one compact versatile unit. The peak torque that can be delivered by each motor after the gear transformation can reach up to $3100 \mathrm{Nm}$. The maximum speed for each motor after the gear can reach up to $189 \mathrm{rpm}$. The robot actuators are equipped with multi-turn absolute encoders to measure the joint positions. The overall structure provides at the traveling-plate level a maximum speed of $2 \mathrm{~m} / \mathrm{s}$ and a maximum acceleration of $4 \mathrm{G}$. The control program of SPIDER4 robot is established within Matlab/Simulink software environment, of MathWorks, and $\mathrm{C} / \mathrm{C}++$ environment which is accessible from Automation Studio software provided by B\&R Perfection in Automation.

2) PIDFF control law: The PIDFF control law is implemented as follows:

$$
\begin{aligned}
\Gamma= & K_{p} e+K_{i} \int e d t+K_{d} \dot{e} \\
& +M\left(q_{d}\right) \ddot{q}_{d}+C\left(q_{d}, \dot{q}_{d}\right) \dot{q}_{d}+G\left(q_{d}\right)
\end{aligned}
$$

with $K_{p}, K_{i}$, and $K_{d}$ are symmetric positive definite matrices representing the feedback gains of the PIDFF controller.

3) Reference trajectories generation: Using linear and circular interpolations, the 3D views of the generated desired trajectories are sketched in Figs. 3 and 4, illustrating the interpolation points within the workspace. The trajectories

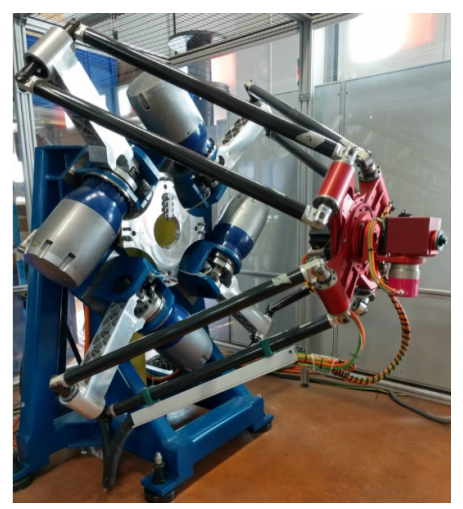

Fig. 2. View of SPIDER4 parallel robot used for real-time experiments. are generated smoothly to reduce the effect of the noise and stimulate all the degrees of freedom of the robot.

4) Performance evaluation criteria: An accuracy evaluation tool frequently used to evaluate differences between a desired trajectory and a measured one is the Root-MeanSquare Error ( $R M S E)$ criterion. The $R M S E$ for Cartesian $\left(R M S E_{C}\right)$ and for joint $\left(R M S E_{J}\right)$ spaces are given respectively as follows:

$$
\begin{aligned}
& R M S E_{C}=\sqrt{\frac{1}{N} \sum_{i=1}^{N}\left(\sum_{j=1}^{m}\left(e_{j}^{2}(i)\right)\right)} \\
& R M S E_{J}=\sqrt{\frac{1}{N} \sum_{i=1}^{N}\left(\sum_{j=1}^{n}\left(e_{j}^{2}(i)\right)\right)}
\end{aligned}
$$

where $N$ denotes the number of the collected samples along the whole trajectory, $e$ represents the tracking error between the desired and measured positions (at Cartesian or joint space), $m$ is the number of translational motions that can be performed by the nacelle of the robot, and $n$ is the number of the actuators.

5) Tuning of the feedback gains: A common method for the tuning of the feedback gains in experiments, often used for complex robotic systems, is the Trial-and-Error method. The gains have been experimentally tuned to get the best tracking performance while avoiding the saturation of the actuators. Increasing further the gains may amplify the noise effect and degrade the tracking performance. The obtained values of the gains resulting from the application of this method are summarized in Table II.

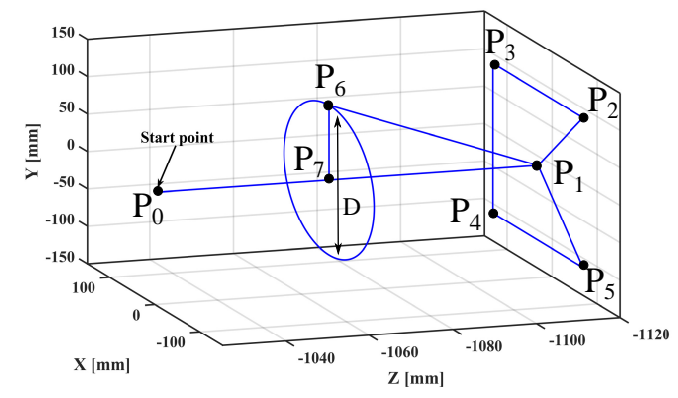

Fig. 3. 3D-view of Trajectory I for SPIDER4 robot in Cartesian space.

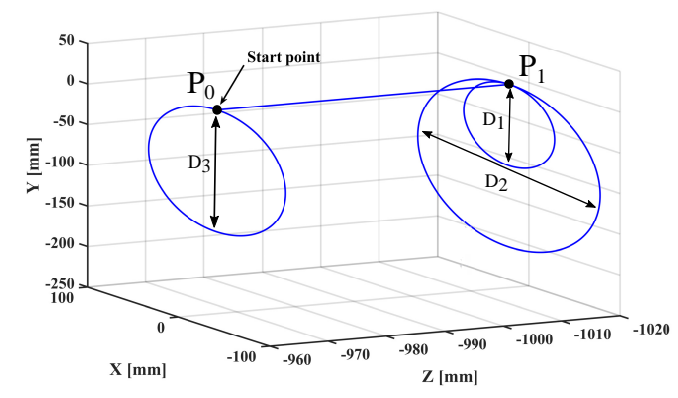

Fig. 4. 3D-view of Trajectory II for SPIDER4 robot in Cartesian space. 
TABLE II

SUMMARY OF THE TUNED FEEDBACK GAINS

\begin{tabular}{ccc}
\hline Standard PIDFF & Conventional CT-ST-SMC & Proposed FF-ST-SMC \\
\hline$K_{p}=3500$ & $\Lambda=80$ & $\Lambda=90$ \\
$K_{d}=40$ & $K_{1}=3$ & $K_{1}=7.5$ \\
$K_{i}=1500$ & $K_{2}=2$ & $K_{2}=5$ \\
& $K_{3}=18$ & $K_{3}=25$ \\
\hline
\end{tabular}

\section{B. Experiment 1: Feedforward PID versus CT-ST-SMC}

In this section, the conventional CT-ST-SMC algorithm and the standard PIDFF control are implemented on SPIDER4 parallel robot. Two scenarios are adopted for this experimental validation: 1) scenario 1: trajectory I shown in Fig. 3, at feedrate of $12000 \mathrm{Inch} /$ Minute, 2) scenario 2: trajectory II shown in Fig. 4, at feedrate of 12000 Inch/Minute.

1) Scenario 1: The conventional CT-ST-SMC algorithm shows a bad behavior when the robot follows Trajectory I. The evolution of the control signals generated by the conventional CT-ST-SMC control law is plotted in Fig. 5. The high chattering effect that appears clearly in the control signals induced a lot of vibrations into the mechanical structure of the robot. The generated control signal may heat the electrical and mechanical parts and lead to premature wear in the actuators. This may be harmful for the actuators and this scenario was not repeated any more.

2) Scenario 2: In this scenario, the robot's nacelle follows Trajectory II at feedrate of 12000 Inch/Minute considering that circular motions can be more smooth on the actuators. The measured signals and the generated output are processed with second order filters only for the conventional CTST-SMC algorithm (due to the above mentioned vibration problem).

The Cartesian tracking errors for both controllers are plotted in Fig. 6. One can observe clearly the superiority of the PIDFF control law w.r.t the conventional CT-ST-SMC in terms of tracking precision along the reference trajectory. The RMSE performance index is evaluated in both Cartesian and joint spaces for both controllers and reported in Table III. The generated control torques of the four motors of SPIDER4 robot for both controllers are depicted in Fig. 7. It is clear that both control algorithms generate an input signal

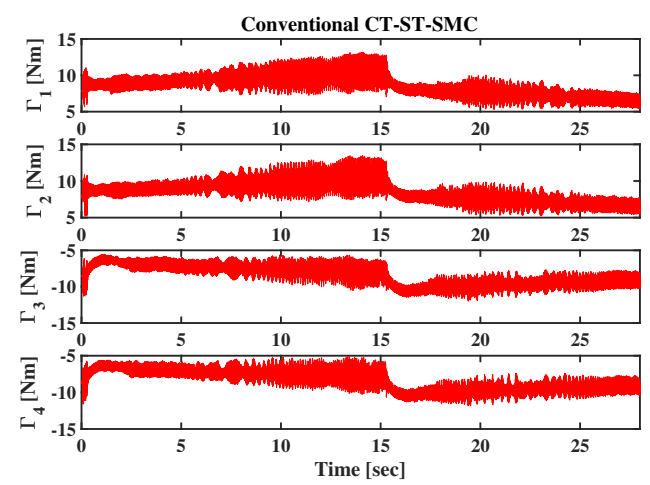

Fig. 5. Experiment 1-Scenario 1: Evolution of the control input torques.
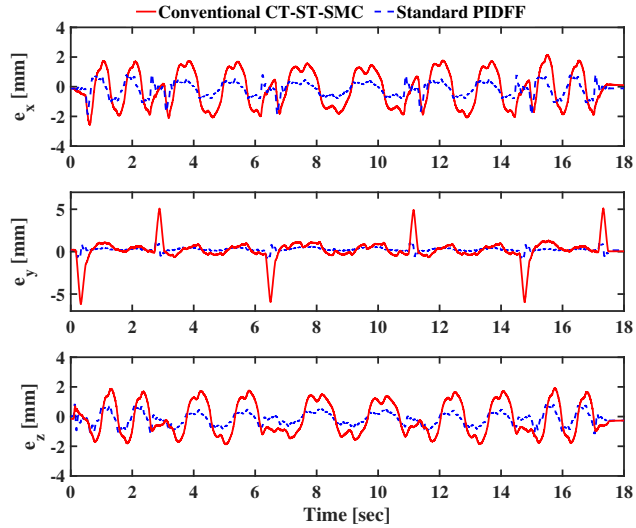

Fig. 6. Experiment 1-Scenario 2: Evolution of the Cartesian tracking errors.

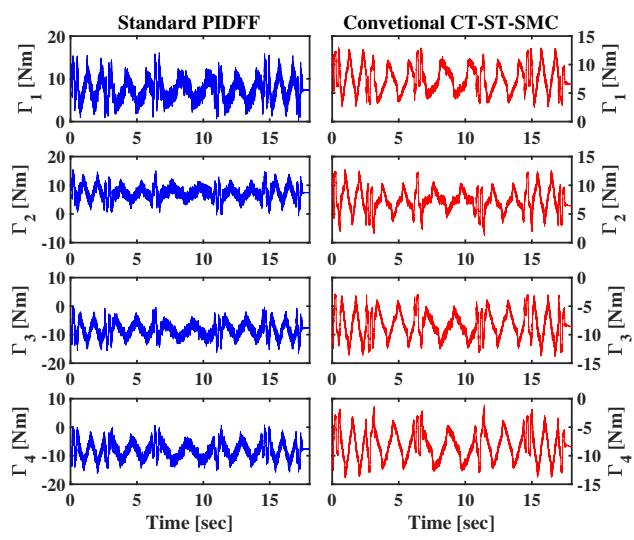

Fig. 7. Experiment 1-Scenario 2: Evolution of the control input torques.

within the admissible limits of the motors. However, still low vibrations can be observed on the mechanical system of the robot with the conventional CT-ST-SMC algorithm.

Finally, this experimental validation proves that computedtorque based control approaches with sliding mode controllers are sensitive to measurement noises and produce chattering effects. This is more effective especially when dealing with dynamical systems of high nonlinearities and large parameter values such as SPIDER4 robot.

\section{Experiment 2: Feedforward PID versus proposed FF-ST-} $S M C$

Within this section, the experimental results on SPIDER4 robot of a PIDFF control and the proposed FeedForward STSMC (FF-ST-SMC) algorithm are presented and discussed. The considered trajectory for these experiments is Trajectory I in which both linear and circular motions are combined. Two main scenarios are also conducted in this experimental

TABLE III

EXPERIMENT 1-SCENARIO 2: CONTROL PERFORMANCE EVALUATION.

\begin{tabular}{ccc}
\hline & $R M S E_{C}[\mathrm{~mm}]$ & $R M S E_{J}[\mathrm{deg}]$ \\
\hline Conventional CT-ST-SMC & 1.9895 & 0.2751 \\
Standard PIDFF & 0.6785 & 0.0521 \\
\hline
\end{tabular}


demonstration: scenario 1: nominal case, scenario 2: robustness towards speed changes.

1) Scenario 1: nominal case: In this scenario, the robot's nacelle has to follow Trajectory I with a feedrate of 6000 Inch/Minute.

Following that trajectory, the Cartesian tracking errors for both controllers are recorded and plotted in Fig. 8. One can observe a good tracking error performed by the proposed controller on all the translational axes compared to the classical PIDFF control. In particular, the tracking error at $\mathrm{y}$-axis is dragged towards zero with the proposed controller by a remarkable compensation that can be noticed clearly in Fig. 8. Due to the horizontal orientation of the kinematic chains of SPIDER4 robot and its heavy parts, the y-axis motion is highly subjected to the effect of gravity. Thus, we can notice from the tracking errors that the proposed FF-STSMC is more robust towards gravitational effects than the standard PIDFF control. The RMSE performance indices in the Cartesian and joint spaces are evaluated for both controllers along the reference trajectory and the obtained values are reported in Table IV. Those indices show a significant improvement of $55.4 \%$ and $44.1 \%$ in both Cartesian and joint spaces respectively.

The generated control torques of the four motors of the robot for both controllers are depicted in Fig. 9. It is clear that both control algorithms generate an input signal within the admissible torques of the motors. Furthermore, both control
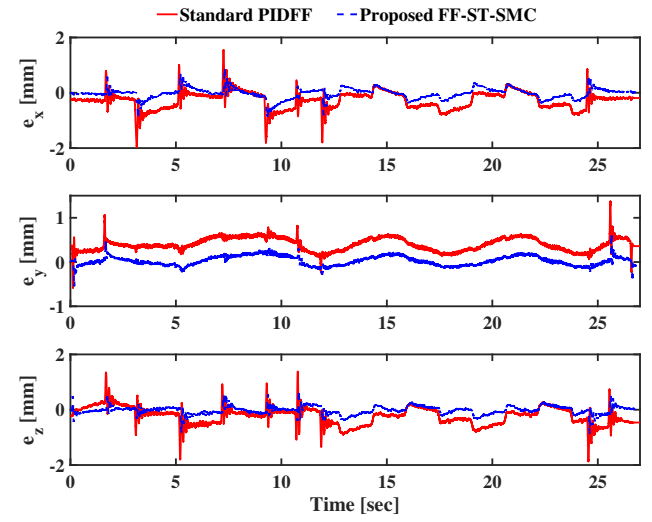

Fig. 8. Experiment 2-Scenario 1: Evolution of the Cartesian tracking errors.
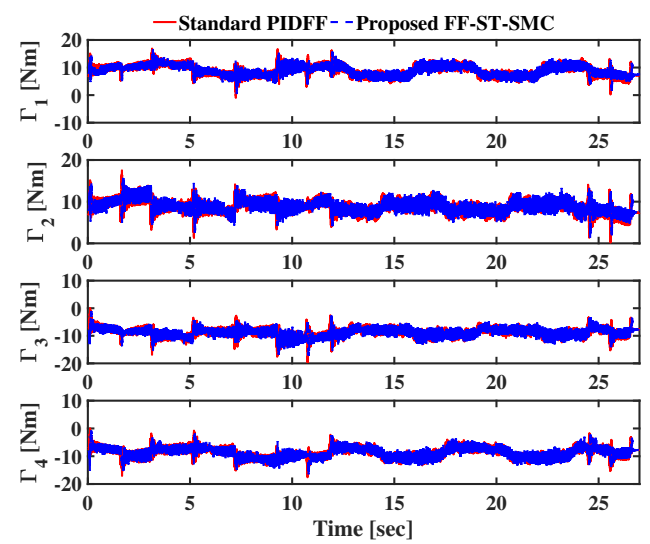

Fig. 9. Experiment 2-Scenario 1: Evolution of the control input torques.
TABLE IV

EXPERIMENT 2-SCENARIO 1: CONTROL PERFORMANCE EVALUATION.

\begin{tabular}{ccc}
\hline & $R M S E_{C}[\mathrm{~mm}]$ & $R M S E_{J}[\mathrm{deg}]$ \\
\hline Standard PIDFF & 0.6026 & 0.0472 \\
Proposed FF-ST-SMC & 0.2689 & 0.0264 \\
\hline Improvements & $55.4 \%$ & $44.1 \%$ \\
\hline
\end{tabular}

signals are smooth enough for the whole trajectory.

This scenario validates the relevance and applicability of the proposed FF-ST-SMC in real-time experiments. It shows also a high dynamic performance of the proposed controller, compared to the classical PIDFF control law.

2) Scenario 2: robustness towards speed changes: In this scenario, the feedrate of the robot's nacelle is increased to 36000 Inch/Minute following reference Trajectory I. The intent behind this scenario is to test the performance of the proposed controller at high-speed motions when the nonlinearity effects of the parallel manipulator increase considerably.

The obtained Cartesian tracking errors for both controllers are depicted in Fig. 10. The dynamic error is considerably reduced for the proposed controller, compared to the PIDFF control law as well as the static error.

The evaluation of the performance indices of both con-
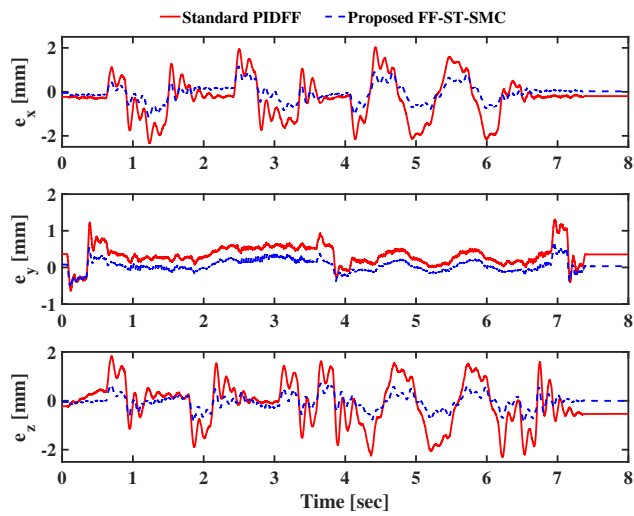

Fig. 10. Experiment 2-Scenario 2: Evolution of the Cartesian tracking errors.

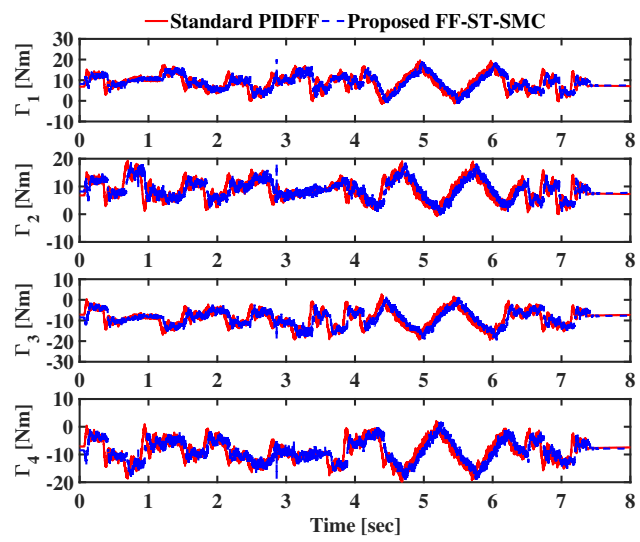

Fig. 11. Experiment 2-Scenario 2: Evolution of the control input torques. 
trollers is reported in Table V. It show an improvement of $44.3 \%$ in Cartesian space and $38.4 \%$ in joint space. The disturbance-rejection and high nonlinearities compensation at high-speed motions are fulfilled by the proposed FF-STSMC approach improving the global dynamic performance of SPIDER4 robot.

The evolution of the control torques generated by both controllers is displayed in Fig. 11. The control signals show a good behavior within the allowable capacities of the motors.

TABLE V

EXPERIMENT 2-SCENARIO 2: CONTROL PERFORMANCE EVALUATION.

\begin{tabular}{ccc}
\hline & $R M S E_{C}[\mathrm{~mm}]$ & $R M S E_{J}[\mathrm{deg}]$ \\
\hline Standard PIDFF & 0.92064 & 0.08421 \\
Proposed FF-ST-SMC & 0.5127 & 0.0519 \\
\hline Improvements & $44.3 \%$ & $38.4 \%$ \\
\hline
\end{tabular}

\section{CONCLUSION AND FUTURE WORK}

A novel model-based ST-SMC strategy based on the feedforward dynamics has been proposed for PKMs. A 5DOF redundantly actuated PKM has been used for realtime validation of the proposed control scheme. The obtained experimental results have shown better global performances of the proposed controller at low and high dynamic operating conditions, compared to conventional computed-torque STSMC and standard PID-FF controllers. Furthermore, it has been experimentally shown the high sensitivity of the conventional computed-torque ST-SMC algorithm to measurements noise, especially when dealing with highly nonlinear PKMs of large dynamic parameter values. As a future work, this paper can be extended with full stability analysis of the proposed control scheme and additional experimental machining scenarios that use the rotational DOFs of the robot.

\section{ACKNOWLEDGMENT}

This work has been supported by the French program Robotex Equipment of Excellence (ANR-10-EQPX-44).

\section{REFERENCES}

[1] A. Chemori, G. Sartori-Natal, and F. Pierrot, "Control of parallel robots: towards very high accelerations," in 10th International MultiConference on Systems, Signals and Devices (SSD), 2013.

[2] H. Cheng, Y.-K. Yiu, and Z. Li, "Dynamics and control of redundantly actuated parallel manipulators," IEEE/ASME Transactions on Mechatronics, vol. 8, no. 4, pp. 483-491, Dec. 2003.

[3] H. Saied, A. Chemori, M. El Rafei, C. Francis, and F. Pierrot, "From Non-model-Based to Model-Based Control of PKMs: A Comparative Study," ser. Mechanisms and Machine Science, R. Rizk and M. Awad, Eds. Cham: Springer International Publishing, 2019, pp. 153-169.

[4] M. Bennehar, A. Chemori, F. Pierrot, and V. Creuze, "Extended modelbased feedforward compensation in 11 adaptive control for mechanical manipulators: Design and experiments," Frontiers in Robotics and AI, vol. 2, p. 32, 2015.

[5] G. Sartori Natal, A. Chemori, and F. Pierrot, "Dual-space adaptive control of redundantly actuated parallel manipulators for extremely fast operations with load changes," in 2012 IEEE International Conference on Robotics and Automation, 2012, pp. 253-258.

[6] H. Jingqing, "Nonlinear PID Controller," Acta Automatica Sinica, vol. 20, no. 04, pp. 487-490, Apr. 1994.

[7] H. Saied, A. Chemori, M. Bouri, M. E. Rafei, C. Francis, and F. Pierrot, "A new time-varying feedback RISE control for second-order nonlinear MIMO systems: theory and experiments," International Journal of Control, vol. 0, pp. 1-14, Dec. 2019.
[8] H. Saied, A. Chemori, M. E. Rafei, C. Francis, and F. Pierret, "Actuator and Friction Dynamics Formulation in Control of PKMs: From Design to Real-Time Experiments," in 2018 IEEE/RSJ International Conference on Intelligent Robots and Systems (IROS), Oct. 2018, pp. 5634-5639.

[9] H. Saied, A. Chemori, M. Michelin, M. El-Rafei, C. Francis, and F. Pierrot, "A Redundant Parallel Robotic Machining Tool: Design, Control and Real-Time Experiments," in New Developments and Advances in Robot Control, ser. Studies in Systems, Decision and Control, N. Derbel, J. Ghommam, and Q. Zhu, Eds. Singapore: Springer, 2019, pp. 39-79.

[10] G. S. Natal, A. Chemori, and F. Pierrot, "Dual-Space Control of Extremely Fast Parallel Manipulators: Payload Changes and the $100 \mathrm{~g}$ Experiment," IEEE Transactions on Control Systems Technology, vol. 23 , no. 4, pp. 1520-1535, July 2015.

[11] M. Bennehar, A. Chemori, and F. Pierrot, "A new revised desired compensation adaptive control for enhanced tracking: application to RA-PKMs," Advanced Robotics, vol. 30, no. 17-18, pp. 1199-1214, Sept. 2016.

[12] G. S. Natal, A. Chemori, and F. Pierrot, "Nonlinear control of parallel manipulators for very high accelerations without velocity measurement: Stability analysis and experiments on par2 parallel manipulator," Robotica, vol. 34, no. 1, pp. 43-70, 2016.

[13] Y. Shtessel, C. Edwards, L. Fridman, and A. Levant, Sliding Mode Control and Observation, ser. Control Engineering. New York, NY: Springer New York, 2014.

[14] M. Zhihong, A. Paplinski, and H. Wu, "A robust MIMO terminal sliding mode control scheme for rigid robotic manipulators," IEEE Transactions on Automatic Control, vol. 39, no. 12, pp. 2464-2469, Dec. 1994.

[15] S. V. Emel'yanov, S. K. Korovin, and A. Levant, "High-order sliding modes in control systems," Computational Mathematics and Modeling, vol. 7, no. 3, pp. 294-318, 1996.

[16] A. Levant, "Sliding order and sliding accuracy in sliding mode control," International Journal of Control, vol. 58, no. 6, pp. 12471263, Dec. 1993.

[17] P. Begon, F. Pierrot, and P. Dauchez, "Fuzzy sliding mode control of a fast parallel robot," in Proceedings of 1995 IEEE International Conference on Robotics and Automation, vol. 1. Nagoya, Japan: IEEE, 1995, pp. 1178-1183.

[18] R. Kumar, A. Chalanga, and B. Bandyopadhyay, "Smooth integral sliding mode controller for the position control of Stewart platform," ISA Transactions, vol. 58, pp. 543-551, Sept. 2015.

[19] M. Bennehar, G. El-Ghazaly, A. Chemori, and F. Pierrot, "A novel adaptive terminal sliding mode control for parallel manipulators: Design and real-time experiments," in 2017 IEEE International Conference on Robotics and Automation (ICRA), 2017, pp. 6086-6092.

[20] A. Hfaiedh, A. Chemori, and A. Abdelkrim, "Disturbance observerbased super-twisting control for the inertia wheel inverted pendulum," in 2020 17th International Multi-Conference on Systems, Signals Devices (SSD), 2020, pp. 747-752.

[21] L. Derafa, A. Benallegue, and L. Fridman, "Super twisting control algorithm for the attitude tracking of a four rotors UAV," Journal of the Franklin Institute, vol. 349, no. 2, pp. 685-699, Mar. 2012.

[22] R. Solea and D. Cernega, "Super twisting sliding mode controller applied to a nonholonomic mobile robot," in 2015 19th International Conference on System Theory, Control and Computing (ICSTCC). Cheile Gradistei, Romania: IEEE, Oct. 2015, pp. 87-92.

[23] S. Mobayen, F. Tchier, and L. Ragoub, "Design of an adaptive tracker for n-link rigid robotic manipulators based on super-twisting global nonlinear sliding mode control," International Journal of Systems Science, vol. 48, no. 9, pp. 1990-2002, July 2017.

[24] J. M. Escorcia-Hernndez, A. Chemori, H. Aguilar-Sierra, and J. A. Monroy-Anieva, "A new solution for machining with RA-PKMs: Modelling, control and experiments," Mechanism and Machine Theory, vol. 150, p. $103864,2020$.

[25] A. Codourey, "Dynamic Modeling of Parallel Robots for ComputedTorque Control Implementation," The International Journal of Robotics Research, vol. 17, no. 12, pp. 1325-1336, Dec. 1998.

[26] C.-S. Jeong, J.-S. Kim, and S.-I. Han, "Tracking Error Constrained Super-twisting Sliding Mode Control for Robotic Systems," International Journal of Control, Automation and Systems, vol. 16, no. 2, pp. 804-814, Apr. 2018.

[27] W. Khalil and E. Dombre, Modeling, Identification and Control of Robots. Butterworth-Heinemann, July 2004.

[28] R. Kelly, V. Santibáñez, and A. Loria, Control of robot manipulators in joint space, ser. Advanced textbooks in control and signal processing. London: Springer, 2005. 